Published in the Proceedings of the Materials

Research Society 1982 Annual Meeting,

November ?-2, 19B2, Boston, MA.

$L B L=-14909$

DE83 003589

\title{
SILICON RADIATION DETECTORS - MATERIALS AND APPLICATIONS*
}

\author{
Jack T. Halton ${ }^{1}$ and Eugene E. Haller ${ }^{1,2}$ \\ TLawrence Berkeley Laboratory and \\ 2Department of Materíals Science \\ University of California \\ Berkeley, Cailifornia 94720 U.S.A.
}

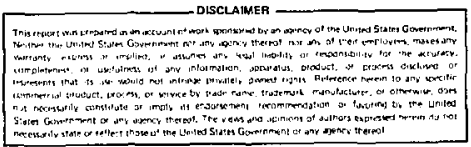

October 1982

\#This work was supported by the Director's Office of Energy Research, Division of Nuclear Physics, and by Nuclear Sciences of the Basic Energy Program, Office of Heaith and Environmental Research of the U.S. Department of Energy under Contract No. DE-AC03-76SF00098. 
JACK T. WALTON1 AND EUGENE E. HALLER 1,2

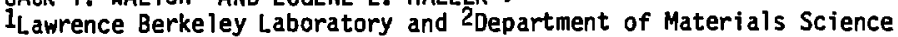
University of California, Berkeley, CA 94720 U.S.A.

\section{ABSTRACT}

Silicon nuclear radiation detectors are available today in a large variety of sizes and types. This profusion has been made possible by the ever increasing quality and diameter silicon single crystals, new processing technologies and techniques, and innovative detector design. The salient characteristics of the four basic detector groups, diffused junction, ion implanted, surface barrier, and lithium drift are reviewed along with the silicon crystal requirements. Results of crystal imperfections detected by lithium ion compensation are presented. Processing technologies and techniques are described. Two recent novel position-sensitive detector designs are discussed-one in high-energy particle track reconstruction and the other in $x$-ray angiography. The unique experimental results obtained with these devices are presented.

\section{INTROOUCTION}

The principal semiconductor materials used in nuclear radiation detector fabrication are silicon and germanium. Germanium has benefited from an extensive program in its application to nuclear radiation detection. Silicon, however, has seen little work in this area. The reasons for this are readily cited:

- Nuclear structure studies which involve the detection of gama rays have prompted the search for high atomic number detector materials and the improvement of germanium purification and crystallography has been a result of this "nuclear" interest. In addition, the lower processing temperature of germanium compared with silicon has permitted the purification and crystal growth of germanium for detector applications with a relatively modest investment.

- Silicon crystals with adequate material characteristics have been available from crystal suppliers to the semiconductor industry at large. However, the silicon processing is usually proprietary and consequently little dialogue has been possible between the detector maker and the crystal manur acturer.

However tenuous the supply of silicon for nuclear detector applications may appear, silicon is used extensively in the fabrication of nuclear radiation detectors. Unlike germanium detectors, silicon detectors can be operated at rocm temperature in many applications. Further, the variety of processing techniques which have been developed in part for integrated circuits fabrication, and the ever increasing size and quality of the silicon single crystals have led to a profusion of silicon detector types.

For the following discussion, let us divide silicon detectors into two classes. We base this division somewhat arbitrarily on the two kinds of stlicon detectors which are typicaliy used in charged particle identifiers (Figure 1) [1]. Thin detectors $(5-500 \mu \mathrm{m})$ in this application, are employed 
to measure the differential energy loss, $\Delta E$, and are commonly called $\Delta E$ detectors. The thicker detectors $(500-5000 \mu \mathrm{m})$ are used to measure the total energy, $E$, and are called E detectors. Algorithms have been developed which relate the differential energy loss, $\Delta E$, and the total energy, $E$, to the particle mass, $M$, and atomic number, $Z$. One algorithm for the generation of a particle identification (P.I.) signal is:

$$
\text { P.I. }=(E+\Delta E)^{n}-\varepsilon^{n} \propto T M^{n-1} Z^{2}
$$

where $T$ is the $\Delta E$ Jetector thickness and $n$ is a coefficient dependent somewhat on the particle ( $n=1.73$ for light ions).

The differences in the physical and electronic requirements on these two detector types, $\Delta E$ and $E$, are reflected directly in the fabrication processes. The $\Delta E$ detectors are normally characterized by:

- Thickness: 5 to $500 \mu m$

- Total volume active (totally depleted)

- Thin contacts on both sides

- Iniform thickness $(+1 y m$ )

whereas the $E$ detectors are characterizad by:

- Thickness $500-5000$ un

- Uniformity not critical

- Thin contact one side

- Partial volume active (partially depleted)

Depending on the specific application, there may be overlaps of the tharacteristic parameters between these two broad detector classes. Thick dutectors have been used occasionally to measure the differential energy loss and, conversely, thin detectors have been used to determine the total particle energy. But for this discussion the soncept of $\triangle E$ and $E$ detectors will aid in illustrating why detector contacts, active volume, etc. are of interest.

In addition to their use at room temperature in charged particle detection, silicon detectors are also used extensively at cryogenic temperatures to detect low energy x-rays $(1-30 \mathrm{keV})$. The detectors employed here are normally thick, i.e., E detectors, since the efficient detection of the photons is of interest.

Finally, in addition to knowing the differential particle energy loss or the particle or photon energy, posicion information is frequently required. There exist many designs for both $\Delta E$ and $E$ position-sensitive detectors, after considering the salient features of $\triangle E$ and $E$ detector fabrication techniques in the following sections, two examples of position-sensitive detectors will be presented.

\section{FABRICATION TECHNIQUES AND DETECTOR CHARACTERISTICS}

Basic semiconductor detector diode structure

for the discussion to follow it will be useful to recall a few basic features of semiconductor diodes [2]. Figure 2 shows a schematic cross section of a planar $n^{+} i p^{+}$diode where the incident radiation, either photons ( $r$, $x$-rays) or charged particles ( $\alpha, \beta$, ions), produces tracks of electron-hole pairs. In the presence of the electric field as shown in the figure, che electron-hole pairs separate and rapidly drift to the contarts. The charge, $Q_{S}$, collected at the contacts is proportional to the energy, $E$, of the inci. dent radiation:

$$
Q_{S}=\frac{E_{c}}{e}
$$

where $c S_{i}=3.6 \mathrm{eV}, c_{\mathrm{Ge}}=2.98 \mathrm{eV}$ and $q=1.6 \times 10^{-19}$ coulcmbs. Further, the rms statistical fluctuation in $\mathrm{Q}_{\mathrm{s}}, \Delta \mathrm{Q}_{\mathrm{S}}$ is: 


$$
\Delta Q_{S}=\left(\frac{E E}{c}\right)^{1 / 2} q
$$

where $F$ is the Fano factor which describes the deviation from normal statistics. The t:ast value currently accepted for $F=0.1$ in both silicon and germanium. Values much greater than this are indicative of signal charge collection problems (i.e., trapping centers) in the detector material.

If a device as shown in fig. 2 is to be an ideal radiation detector, the following set of requirements have to be met: a) the sensitive region must be free of charge trapping centers which would affect the amount of charge collected; b) the contacts need to be thin enough to allow the passage of radiation without appreciable energy or intensity loss; c) the contacts need to remain non-injecting for reverse bias field strengths of $E=10^{2}$ to $10^{4} \mathrm{Vcm}^{-1}$; d) the surfaces have to be passivated against detrimental effects from the ambients; and e) the noise which is caused by the reverse leakage current has to be kept smali compared to the sigrial fluctuations. In the case of $x$-ray detectors, this leads to the requirement of cooling the detectors.

The signal charge, $Q_{s}$, produced by nuclear radiation and the rms fluctuation in this charge, $\Delta Q_{s}$ (Eqs. 2 and 3 ), as a function of energy are shown in Fig. 3. Also indicated in the figure are representative values of noise levels for a silicon detector and amplifying electronics achieved at room temperature and at cryogenic temperat'ses. The room temperature noise leve] is caused by shot noise resulting from a detector leakage current of abaut 5 uA.

As shown in Fig. 2, there are two contacts on the detector, one of which is "rectifying" and the other which is often called a "blocking" contact*. In the case where the electric field lines from the "rectifying" contact reach through to the "blocking" contact (as in the case of the $\Delta E$ detectors) this "blocking" contact must inhibit the injection of minority carriers into the active volume of the detector. The formation of this "blocking" or "non-injecting" contact is as important for $\triangle E$ detectors' successful operation as the fabrication of the rectifying contact. With $E$ detectors, the detector volume is frequently not totally active and consequently, the field lines from the rectifying junction may not reach the opposite contact. The requirement of full depletion of $\Delta E$ detectors and only partial depletion of $E$ detectors and the resulting differences in contact properties and formation are the most distinguishing features between the two groups of detectors.

There are four basic technologies used to fabricate silicon $\Delta E$ and $E$ detectors--three are related to contact formation, surface barrier (or metal Schottky barrier) diffused junction, and ion implantation, while the fourth lithium ion compensation, is concerned with the modification of the silicon material properties to allow wide depletion regions. Semiconductor detectors are frequently labeled by the dominant technology used in their fabrication even though more than one technology may be used. For example, the fabrication of It thium fon compensated diodes employs both diffusion and surface barrier technology. In the following sections the four basic detector types are separately reviewed. Special techniques employing epitaxial regrowth and multiple layered doped regions have also been explored, but they will not be discussed here.

*With an $n^{+} p^{+}$structure, the $n^{+}$is the rectifying contact and $\mathrm{p}^{+}$the blocking one. With an $n^{+} \cup p^{+}$the $n^{+}$is blocking and the $p^{+}$is rectifying. The symbols and $u$ refer to high resistivity $p$ and $n$ material respectively. In the limit of ultra-pure or perfectly compensated material this distinction regarding the contacts vanishes. 
Surface barrier detectors

A schenatic of a silicon surf ace barrier detector and its holder is shown in Fig. 4. The following preparation steps are used to fabricate such a detector [3]. The silicon wafer after a series of chemica] etching and surface preparation steps is potted in freshly mixed epoxy which has been painted onto a mounting ring*. The epoxies employed are elther $n-t y p e$ (aliphatic amines) or p-type (catalytic) in their effects on the silicon surfaces. The details on how these epoxies affect the silicon surfaces and what influence the prior chemical treatment of the silicon wafer has on the resultant device performance are still not well understood. However, procedures have evolved which do allow the successful production of these detectors with the cured epoxy providing both the required junction protection and the machanical adhesion to $a$ mounting ring.

After curing the epoxy at $50-60^{\circ} \mathrm{C}$, metal contacts $(100-200 \AA$ thick) are evaporated over the epoxy and silicon surfaces. On the device shown in the figure, the aluminum forms the "rectifying" contact on the p-type silicon, while gold forms the noninjecting or "blocking" contact. With n-type surface barriers, the roles of these metals are reversed.

The contacts which are formed by evaporation of metals onto silicon surfaces which have been exposed to various oxidizing environments, do not adhere very strongly to the silicon. Consequently, the metal Schottky barrier contacts are easily damaged. Such damage can strongly affect both the rectifying and blocking properties of the contacts.

The silicon used in the fabrication of these detectors is extremely pure in comparison with that employed in the semiconductor electronics industry. For silicon the depletion region width, $x_{0}$, is given by [4]:

$$
x_{0}(\mu m)=3.59 \times 10^{-3}\left(\frac{v}{N}\right)
$$

where $N$ is the net impurity concentration $\left(\mathrm{cm}^{3}\right)$ and $V$ is the applied voltage (V). Figure 5 shows the depletion region width with 100 volts bias as a function of the net impurity concentration. From this figure, it is seen that the net impurity range of interest for $\mathrm{AE}$ detector fabrication is $[\mathrm{N}] \leq 10^{15} \mathrm{Cm}^{3}$ and for $E$ detectors is $[\mathrm{H}] \leq 3 \times 10 \mathrm{I}_{\mathrm{CT}} \mathrm{T}^{3}$.

The leakage current assōciated with a reverse biased semiconductor junction has three components: diffusion (or thermionic emission in the case of surface barriers), thermal generation and surface leakage. For silicon detectors operated at room temperature (300K) and lower, the diffusion component is so small that it can be ignored. The surface leakage current is strongly dependent on the fabrication technique, while the thermal generation current, $i_{g}$, at room temperature is given by [4]:

$$
i_{g}=\frac{0.128 x_{0}}{\tau} \mu \mathrm{Acm}^{-2}
$$

where the depletion depth, $x_{0}$ is measured in $\mu m$ and $\tau$ is the minority carrier lifetime in usec. From this it becomes clear that the numerical value of the minority carrier lifetime should be comparable to the numerical value of the detector thickness if the generation current is to be maintained at the $\mu A$ level. High quality slificon crystals typically exhibit lifetimes of $\tau>500 \mu \mathrm{sec}$. Since the surface barrier detector fabrication does not involve

*Whlle the figure shows a p-type siticon wafer, the more common commercially available surface barriers are made on n-type silicon. 
any high temperature processing steps which could degrade the lifetime, the lifetime in the final detector should be that of the starting crystal and conse quent ly the generation current should be less than $1 \mu \mathrm{Acm}^{2}$.

The surface barrier detector material requirements and device characteristics are tabulated in Table I. Surface barrier detectors are fairly readily fabricated and have exceptionally thin contacts. They are, however, relatively fragile and are nomally limited to readily machinable geometries.

\section{Diffused function detectors}

Silicon planar diffused junction technology employing either $n$ - or p-tyee silicon has been utilized for many years in the fabrication of silicon radiation detectors [5]. Figure 6, which shows a detector made on p-type silicon, indicates the features of these devices. The silicon dioxide grown at 950 $1000^{\circ} \mathrm{C}$ and $3000-7000 \mathrm{~A}$ thick provides the junction diffusion masl: and edge protection. Unlike the epoxy-silicon interface of the surface barrier detector, the properites of this silicon-silicon dioxide interface are fairly well understood [6]. The $\mathrm{n}^{+}$and $\mathrm{p}^{+}$contacts are diffused $0.2-0.5 \mu \mathrm{m}$ deep (typically at $900-950^{\circ} \mathrm{C}, 10$ - 30 minutes) into the wafer and consequently are much more rugged than the metal contacts on the surface barrier detectors. The diffused contacts are, however, thicker than the metal barriers, and for many applications this is a serious limitation. In addition, the high temperature processing required for the growth of the silicon dioxide often causes a significant decrease in the minority carrier lifetime. This results in the generation current ( $\left.E_{q} .5\right)$, which is inverse?y proportional to the lifetime, being larger than that obtained with the surface barrier technology. However, the recently reporteo use of chlorine during the silicon dioxide growth has indicated that the minority carrier lifetime can be maintained and consequently the leakage currents reduced to values approaching that achieved with surface barrier detectors [7].

The processing of diffused junction detectors is complicated both in the procedures and equipment required in comparison with the surface barrier technology. However, diffused junction detectors are very rugged and the planar technology allows the ready use of photolithographic techniques which permit a wide variety of detector geometries to be produced. The diffused junction material requirement5, commonly employed diffusants and detector characteristics are given in Table I.

Ion implanted detectors

Ion implantation is also used to fabricate the contacts on silicon radia$t$ tion detectors. This technique offers the advantages of precise controt of the depth distribution of the doping impurities and iower temperature processing in forming the contacts. Figure 7 indicates the features of a detector made on p-type silicon with ion implanted contacts. The silicon dioxide, as shown, again forms a mask and passivates the surface around the rectifying junction.

Processing nomaliy involves the growth of silicon dioxide, as previously, followed by the jimplantation of the $n^{+}$and $p^{+}$contacts $(10-30 \mathrm{keV}, 2-$ $5 \times 10^{12}$ ions $\left.\mathrm{cm}^{2}\right)$. The radiation damage produced by the implantation is then annealed ( $700-800^{\circ} \mathrm{C}, \mathrm{J} .0$ - 30 minutes). Ion implanted contacts exhibit thicknesses of the order of 300 to $1000 \AA$.

The ion implantation process does not require the same care which is needed for the diffusion process since the activation of dopants occurs at Tower tcmperatures than those needed for diffusion. The doping impurities are introduced at room temperature or lower with vary precise control. The equipment requirements are, however, greater. The use of ion implantation has resulted in devices which are rugged yet possess thin entrance contacts. The 
comwon dopants, material requirements and characteristics of these devices are also presented in Table I.

Lithium ion compensated detectors

While siticon crystals are avallable with resistivities which would allow the fabrication by the preceding techniques of detectors greater than $1 \mathrm{~mm}$ in depletion depth, these crystals are presently expensive and scarce. Consequently, lithium ion compensation is extensively used to produce the thicker detectors. The following procedure is used for theli fabrication. Lithium is diffused into the p-type wafer (nominal 1000 ohm-cm) forming the $\mathrm{n}^{+}$contact. A groove is then cut into the wafer using an ultrasonic cutter. This groove and the $p^{+}$contact region are chemically etched with the resultant device as shown in Fig. 3 . The $p^{+}$contact is formed by evaporating gold onto the elched region. After surface treatment to minimize the leakage currents, the device is put onto "drift". The "drift" consists of applying a reverse bias $\left(500-1000\right.$ volts) and heat $\left(110-150^{\circ} \mathrm{C}\right)$ to the device. This causes the 1 ithium ions to drift under the influence of the applied electric field from the $\mathrm{n}^{+}$contact into the bulk p material. The 1 ithium ions, which are interstitial, compensate the nascent boron impurities to produce a nearly intrinsic material in the compensated region.

Lithium ion compensation has been used for many years in both silicon and gemanium detector fabrication with varying degrees of success. A major obstacle to successful lithium ton compensation has, historically, been the presence of oxygen in the silicon and germanium crystals. Oxygen forms an immobile donor complex with lithium which leads, for medium oxygen concentrations $\left(\left[\mathrm{O}_{2}\right]=10^{15} \mathrm{~cm}^{3}\right)$, to a reduction of the 1 ithium ion mobility and in the case of high oxygen concentration $\left(\left[0_{2}\right] \geq 10^{17} \mathrm{~cm}^{-3}\right)$, to a complete immobilization of the lithium for all practical purposes [8].

Another interference in the drift process has been studied more recently. It is related to microdefects ( $A$ - and B-type swirls) which can be present in large diameter, dislocation-free floating-zone crystals. The generation and distribution of the $A$ and $B$ swirls has been associated with the crystal growth conditions and crystal growing procedures have been advanced to reduce or eliminate their generation. Further, it is believed that $A$ swirls, which consist of dislocation loops surrounding interstitial sllicon atom platelets, evolve from B swirls which are partial loops [9].

We have on occasion encountered dislocasion--free silicon crystals in which the lithium ion mobility was reduced in spite of the fact that the oxygen concentration was low (i.e., $\left[0_{2}\right]<10^{15} \mathrm{~cm}^{3}$ ). Consequent ly, we initiated a study to detemine whether the $A$ and $B$ swirls which are affected directly by the growth conditions of dislocation-free silicon were responsible for the observed lithium ion mobllity and/or the charge collection in the completed detectors.

Our study [10] consisted of five 1000 - 2000תcm p-type crystals which had been grown under differant floating zone conditions. Two crystals were grown with a relatively large molten zone, while the remaining three were grown with a smaller zone (the large zone was $50 \%$ greater than the small one). The 1 ithium compensated detectors made from these crystals were scanned with an aloha source with various applied bias voltages. The results of these alpha scans are sriwn in Fig. 9. It is readliy apparent that there is a marked difference in the low bias charge collection propertles of these detectors. The detectors made from the two crystals with the larger molten zone have constant charge collection response, whereas those made from the crystals with the smaller zone show pronounced dips in the central region. We believe that this charge collection deficiency as shown in Fig. 9 is related to the formation of micro defects (pre B swirls) during the crystal growth. Further, we believe that if these defects are present in sufficlent density, the lithium 
ion mobility will be reduced. This technique of examining 1 ithium compensated detector charge collection performance with low bias voltages appears to be very sensitive to the presence of micro defects in the crystal. While this has proven useful in assessing the quality of sllicon for detector fabrication, it may also be useful in correlating crystal growth conditions with defect formation.

The silicon crystals employed in the lithium ion compensation technique are of the floating zone $k$ ind, $p$ type with 1000 - $2000 \Omega \mathrm{cm}$ resistivity (corresponding to $[B]=1013^{\mathrm{cm}} \mathrm{m}^{3}$ ) and $500-1000_{\mu} \mathrm{sec}$ minority carrier lifetime. The resistivity range is 1 imited at high boron concentrations ([B] $\geq 10^{14} \mathrm{~cm}-3$ ) by the reduced lithium ion mobility due to lithium boron pair formation. Low boron concentrations ( $[\mathrm{B}] \leq 5 \times 10^{1} \mathrm{~cm}^{3}$ ) make it difficult to control the silicon surface states and, consequently, the leakage currents during the ion drift process.

The presence of dislocations also affects the lithium ion mobility [11]. Consequently, we normally use crystals having a dislocation density between 0 and $4000 \mathrm{~cm}^{2}$. However, we noted in our previously mentioned work on dislocation-free silicon crystals that the presence of "pre- $B$ " type swirls in suff $i-$ cient density can also reduce the lithium ion mobility. Therefore, the specification of zero dislocations may not necessarily guarantee an adequate lithium ion mobility in the crystal.

The specification of a minority carrier lifetime of $500-1000 \mu \mathrm{sec}$ is intended to select crystals which have a sma11 deep trap concentration. Deep traps affect the extremely stringent charge collection properties of nuclear radiation detectors. Mayer [12] has shown that for the trapping effects to be negligible, the mean time that a carrier must be free before being trapped. $\tau^{+}$, is related to the detector thickness, $L$, by:

$$
\tau^{+}=\frac{100 \mathrm{~L}}{v_{d}}
$$

where $v_{d}$ is the carrier drift velocity, $-1 \times 10^{6} \mathrm{cms}^{-1}$, in the usial lithium drift detector at roon temperature. For a $5 \mathrm{~mm}$ thick detector, $\tau^{+}=50 \mu \mathrm{sec}$. The Darameter $\tau^{+}$is a detector parameter and is not the minority carrier lifetime which is measured at near equilibrium conditions. The selection of 500 $1000 \mu \mathrm{sec}$ for the minority carrier lifetime specification avoids any charge collection problems which could be due to this crystal parameter.

While the oreceding discussion has centered on room temperature applications of these detectors, lithium drifted detectors are also used extensively at cryogenic temperatures to detect soft $x$-rays $(E=1-30 \mathrm{keV})$. In these applications, trapping centers which are inactive at room temperature become much more critical. However at the present time, there is little understanding of the nature and density of the traps and their influence on the charge collection processes at these low temperatures. Fano factors for lithium compensated silicon detectors of the order of 0.1 , which is indicative of low trap density (see discussion regarding Eq. 3), are commonly obtained so that the anomalous crystals have not yet warranted investigation.

The junction edge protection on lithium drifted detectors is frequently accomplished by coating the surfaces with paints, varnishes or other materials which do not substantially alter the detector surface states. The exact formulation of these coating materials employed by the various detector manufacturers is proprietary as it has normally been developed over a long series of trials and evaluations.

The lithium compensation can be done with little equipment and the process itself is comparatively simple. Passivation of the device surfaces, however, borders on being an art and consequently the long term stability of these devices, especially at room temperature, is variable. Navertheless these detectors are used extensively in $\Delta E$, $E$ telescopes and in $x-r a y$ detector systems. The main features of these devices are also given in Table I. 
In choosing the folowing examples to illustrate silicon detector capabilitfes, we attempted to select a few applications which not only indicate what has been accomplished, but which could also point out areas of future development.

\section{$\Delta E, E$ telescopes}

Detector telescopes employing $\Delta E, E$ dual arrangement as shown earlier in Fig. 1 or having mult fole (three or more) detectors have been used for many years in the identification of nuclear particles. In the $\Delta E$, $E$ dual telescope a single identification is made using an algorithm such as that of Eq. 1. By employing a second $\Delta E$ detector and performing two identifications on the same particle passing through the telescope, the signals can be compared and anomalous events rejected. The performance of a dual telescope in measuring the products from $129 \mathrm{MeV}$ alpha part icles impinging on a $12 \mathrm{C}$ target are shown in Fig. 10a, while the improvement obtained by employing a $\triangle E, \Delta E, E$ triple telescope during the same experiment is evident in Fig. 10b [13]. The particle identifier techniques have been extended to multiple-element detector telescopes which have been used to study cosmic ray composition [14] and neutron rich light nuclei stability [15]. In these latter applications, lithium ion compensated detectors with areas of 15 to $40 \mathrm{~cm}^{2}, 3$ to $5 \mathrm{~mm}$ thick and 8 to 16 detectors in a telescope have been employed to measure the high energy, low intensity particles. Detectors of this area and thickness place very heavy demands on the silicon crystal quality and the future availability of such large area detector telescopes is very dependent on the interest and ability of crystal suppliers to provide these large diameter, high-purity silicon crystals. We have indicated previously the difficulties in lithium ion conpensation with these large diameter crystals and additional work is required in relating the crystal yrowth conditions for these large crystals with the resultant detector characteristics.

\section{Position-sensitive detectors}

It was noted earlier that in addition to the particle energy, its position is also frequently of interest. Two basic types of semiconductor positionsensitive detectors are in use--cont inuous element and discrete element [16]. Cont inuous-element detectors use a resistive layer to generate the position signal. 0i screte-element detectors have a series of what may be considered individual detectors on a common wafer. The following two examples are discrete-element devices, the first being used in high-energy physics and the second in nuclear medictne.

Particle track reconstruction: With the new high-energy physics acceierators proposed or under construction, there is a growing awareness that new generation of particle detectors is required to adequately utilize the capabilities of these machines. Among the new detectors proposed are arrays of silicon wafers $100-300 \mu m$ thick, each having $50-500$ equally spaced stripes (10 -

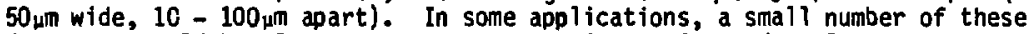
detectors would be placed near the interaction region and would be used to project the particle tracks back into the interaction region or vertex where short-lived, chanmed-particle decay (which is of interest) occurs.

A prototype of one of these vertex detectors is shown in Fig. 11 . The detector is a $150 \mu \mathrm{m}$ thick diffused junction device having 48 , $1 \mathrm{~cm}$ iong stripes, $20 \mu m$ wide and $60 \mu m$ apart.

The preliminary resuits obtained in a test at the Femi Laboratory indicated that a five-detector telescope of these detectors was successful in particle track reconstruction [17]. Similar results have been obtained earlier 
at CERN [18] and we expect that detectors of this type will be in growing derand especially with the newer accelerators. There exists, however, a readout problem. The detectors tested at the Fermi Laboratory have one amplifier chain connected to each stripe. For experiments involving larger area devices and/or devices with a higher density of stripes, the electronics costs of this approach may become prohibitive. Consequently, development of these vertex detectors integrated with the required electronics or a ready hybridization arrangement appears to be of paramount importance.

$x-$ ray angiography: Angiography [19], as currently employed, involves the visualization of blood vessels by use of the $x$-ray absorption characteristics of iodine-containing compounds which are injected directly into the artery of interest. Conventional $x$-ray sources provide a broad spect,rum of energies and consequently, a relatively high dosage of iodine is required to obtain adequate images. For many individuals, the iodine concentration required and its injection by means of a catheter oresent serious risks. However, the availability of high-intensity $x$-rays produced by synchrotron radiation at SLAC allows the monochromatization of these $x$-rays with sufficient intensity that images can be produced with much lower concentrations of iodine and non-catheterization. The essence of the technique is shown in Fig. 12 where the monochromatization of synchrotron radiation by Bragg diffraction is indicated. The $x$-ray energy is switched above and below the $K$ absorption edge of jodine to allow the subtraction of the background. While a 256-element detector is shown in the figure, preliminary measurements have, in fact, been performed with a 30-element detector shown schematically in Fig. 13. Results obtained on the blood flow in a dog's heart are shown in Fig. 14. Development of larger area detectors with smaller element size is now under way with tie prospect of achieving high-quality images on heart patients as the immediate goal.

\section{SUMMARY}

Silicon radiation detectors have been examined principally in relation to their use in charged-particles telescopes. The four basic fabrication techniques, surface barrier, diffused junction, ion implantation and lithium ion compensation have been reviewed.

While silicon detectors are widely used in scientific research, the silicon crystals used to fabricate these detectors are produced by companies whose principal interest is supplying silicon to the semiconductor industry. Consequently, the silicon supply for detector fabrication is often tenuous. However, given tile availability of high quality silicon the examples given denonstrate the flexibility of silicon detectors both in fabrication techniques and in final applications. Furthermore, the continued interest by the semiconductor industry in silicon processing will undoubtedly yield additional processing advances which will present even greater flexibility in silicon detector designs and uses.

\section{ACKNOWLEOGMENTS}

We appreciate the continued interest and comments of $F$. S. Goulding in regard to silicon semiconductor research and development. This review has also benefited from discussions with $W$. L. Hansen, R. H. Pehl, A. C. Thompson, H. A. Sormer and $Y$. Wong at LBL, E. B. Hughes at Stanford University and G. R. Kalbfleisch at the University of Oklahoma.

This work was supported by the Director's Office of Energy Research, Division of Nuclear Phys'..s, and by Nuclear Sciences of the Basic Energy Program, Office of Health and Eivironmental Research of the U. S. Department of Energy under Contract No. DE-ACO3-76SF0009B. 


\section{REFERENCES}

1. F. S. Goulding and B. G. Harvey, Annual Review of Muclear Science 25, 167 (1975).

2. E. E. Haller, IEEE Trans. Nucl. Sci. NS-29, No. 3, 1109 (1982).

3. R. C. Tramme11, IEEE Trans. Nuc1. Sci. NS-25, No. 2, 910 (1978).

4. A. Coche and P. Siffert in: Semiconductor Detectors, G. Bertol ini and $A$. Coche eds. (North-Holland, Amsterdam 1968) Ch. 2.

5. F. S. Goulding, Nucl. Instr. and Meth. 43, 1 (1966).

6. B. E. Deal in: Semiconductor Silicon 1977, H. R. Huff and E. Sirtl eds. (The \&lectrochemical Society, Princeton 1977) p. 276.

7. J. Kemmer, Muc1. Instr. and Meth. 169, 499 (1980).

8. P. Siffert and A. Coche in: Semiconductor Detectors, G. Bertolini and A. Coche eds. (North-Holland, Amsterdam 1968) Ch. 1.

9. A. J. R. DeKock in: Handbook on Semiconductors, Vol. 3, S. P. Keller ed. (North-Holland, Amsterdam 1980) Ch. 4.

10. A. Fong, J. T. Walton, E. E. Haller, H. A. Sommer and J. Guldberg, Nucl. Instr. and Meth. 199, 623 (1982).

11. H. J. Guislain, W. K. Schoenmaeker and L. H. DeLaet, Nucl. Instr. and Meth. 101, 1 (1972).

12. J. H. Mayer in: Semiconductor Detectors, G. Bertolini and A. Coche eds. (North-Holland, Amsterd3m 1968) Ch. 5.

13. J. Cerny, S. W. Casper, G. W. Bulter, H. Brunnader, R, L. McGrath and F. S. Goulding, Nucl. Instr. and Meth. 45, 337 (1966).

14. M. E. Wiedenbeck and D. E. Greiner, The Astrophysical Journal 239. L139 (1980).

15. T. J. M. Symons in: Atomic Masses and Fundamental Constants, J. Nolen and W. Benenson eds. T(Plenum Press, 1980) D. 61 .

16. E. Laegsgaard, Nucl. Instr. and Meth. 162, 93 (1979).

17. G. R. Kalbfleisch, to be published in Nucl. Instr. and Meth.

18. E. H. M. Heijne, L. Hubbeling, B. D. Hyams, P. Jarron, P. Lazeyras, F. Pinz, J. C. Vermeulen and A. Hylie, Nuci. Instr. and Meth. 1\%8, 331 (1 ys0).

19. A. C. Thompson, F. S. Goulding, H. A. Sommer, J. T. Walton, E. B. Hughes, J. Rolfe and H. D. Zennan, IEEE Trans. Nucl. Sci. NS-29, No. 1, 793 (1982). 
TABLE I.

Detector Type Parameter Surface Barrier Diffused

Lithium Parameter

Resistivity $(\Omega \mathrm{cm})$ $50-50,000$ $50-50,000$ Ion Implanted

Lifetime ( $\mu \mathrm{sec}$ )

$>100$

$>100$ $50-50,500$

$n, F$ $>100$

$1000-3000$

Type - Floating Zone

n, p

$n, p$

$>\infty 00$

EPD $\left(\mathrm{cm}^{-2}\right)$

$<4000$

$<4000$

$<4000$

D

$\mathrm{n}^{+}$Contact

A1

$P$, As

$P$, AS

$<4000$

$p^{+}$Contact

$\mathrm{Au}, \mathrm{Pt}$

B

B

Li

$\mathrm{Pa}, \mathrm{Cr}, \mathrm{Ni}$

$-100-150$

$-60-80$

Au, $\mathrm{Pt}$

$-20-40$

Contact Thi
(ugmcm

Thickness Range ( $\mu \mathrm{m})$

5-1000

$5-1000$

$5-1000$ $\mathrm{Pd}, \mathrm{Cr}, \mathrm{Ni}$

Area $\left(\mathrm{cm}^{2}\right)$

$<30$

$<20$

$<30$

$\mathrm{p}^{+}-20-40$

$\mathrm{n}^{+}-200 \rightarrow$

$3 \times 10^{4}$

500-5000

$<40$ 


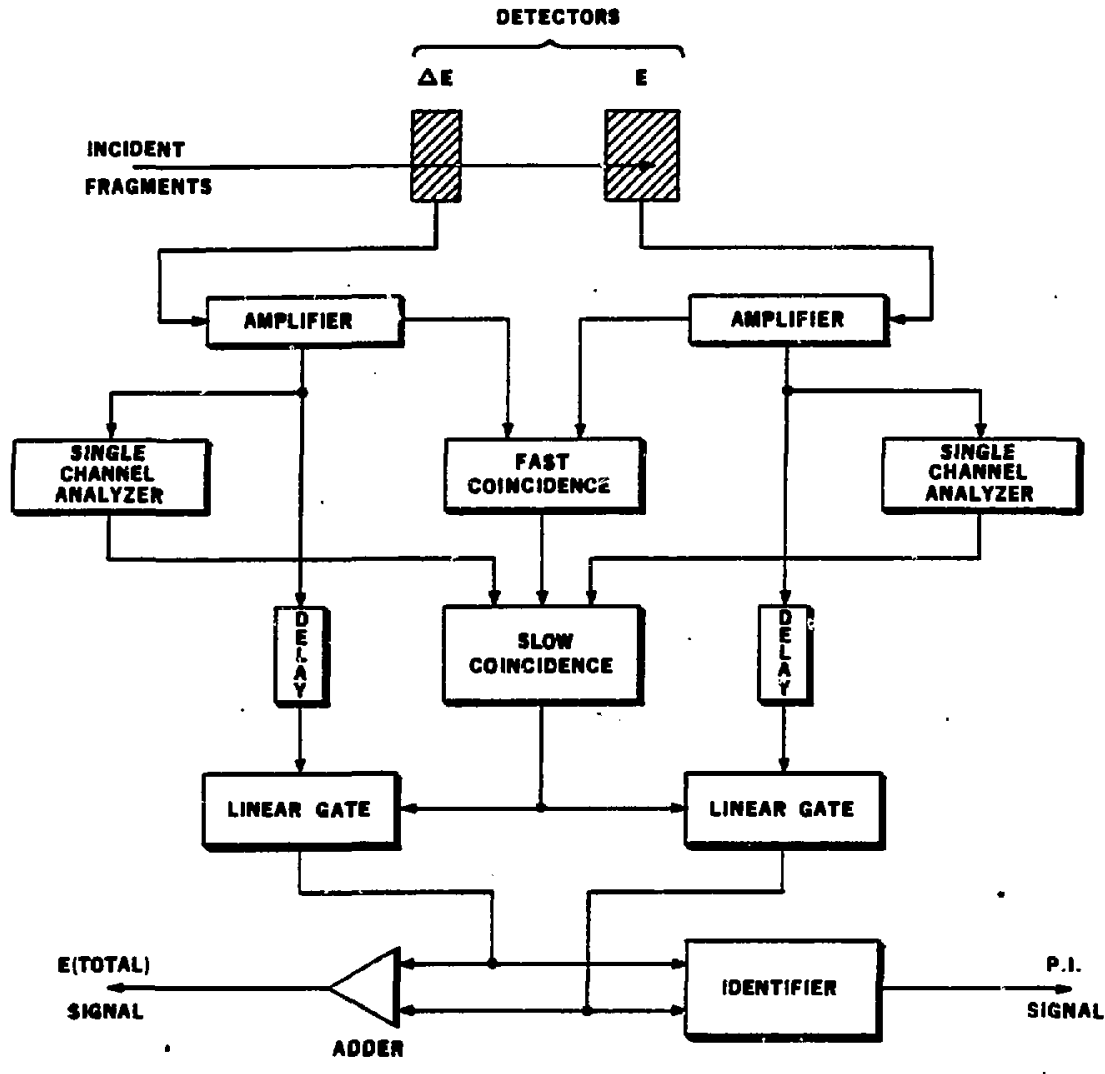

XBL 741-93

Fig. 1. Block diagran of the $\triangle E, E$ detector telescope particle identifier system iypicaliy employed to study nuclear reaction products. 


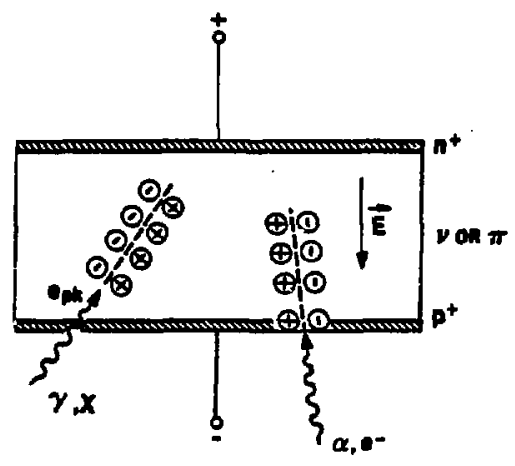

xac $210.27+3$

Fig. 2. Schematic cross secion of a nuclear radiation detector. The contacts are labeled $n^{+}$and $p^{+}$while the bulk material is labeled $v$ and $x$ corresponding to high resistivity $n$ or $p$ silicon respectively.

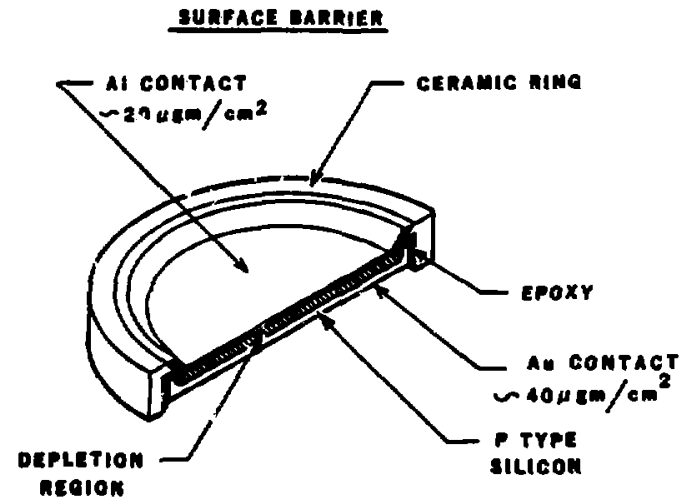

xat $792-8270$

Fig. 4. Mechanical details of surface barrier detector fabrication. 


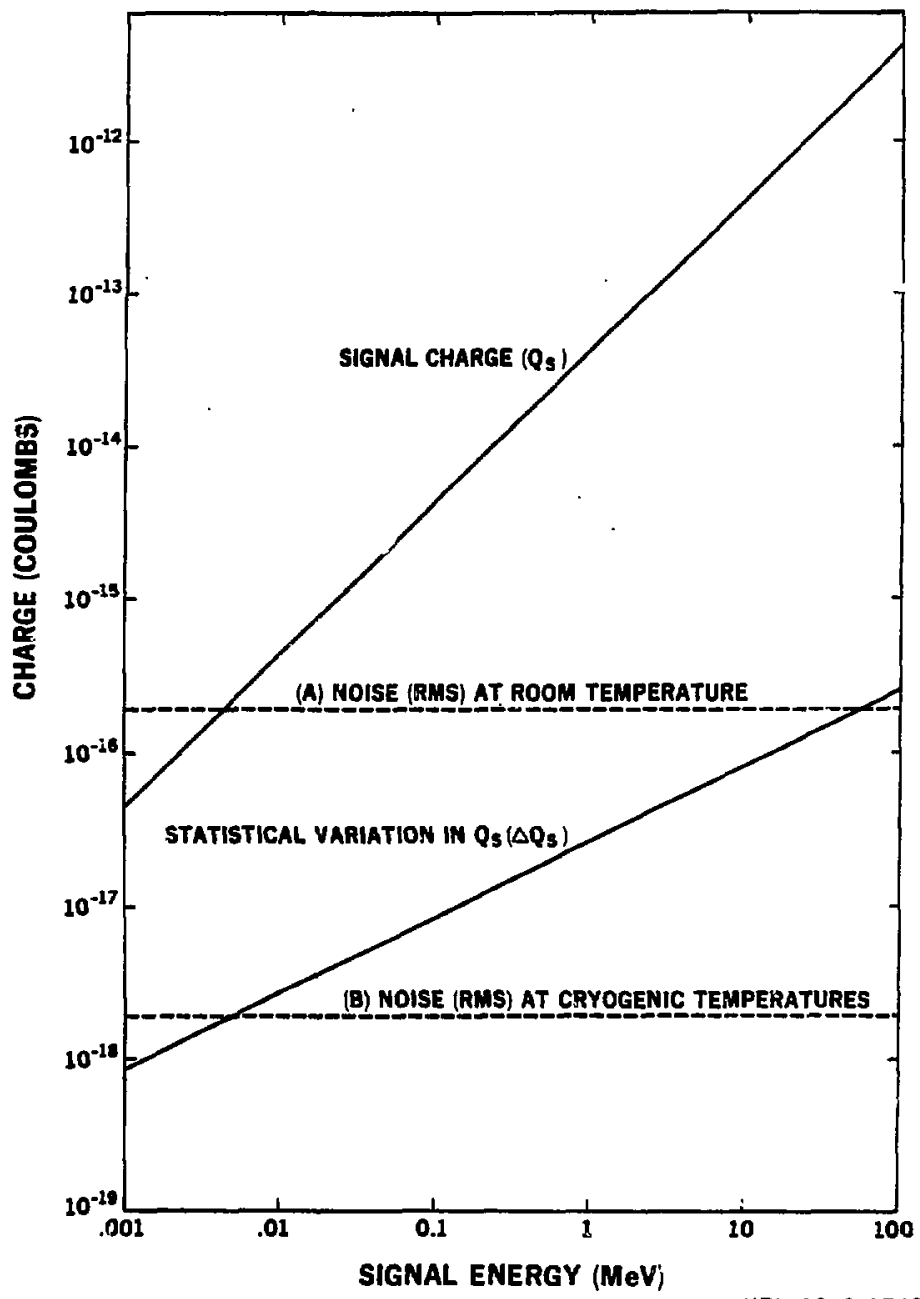

XBL $8210-2742$

Fig. 3. Signal charge, $Q_{s}$, (coulombs) produced by incident signal radiation (MeV) and the statistical variation, $\mathrm{BQ}_{S}$, in this signal charge for silicon nuclear radiation detectors. The noise of a representative detector and electronics at room temperature $(A)$ and at cryogenic teiperatures (B) are also shown. 

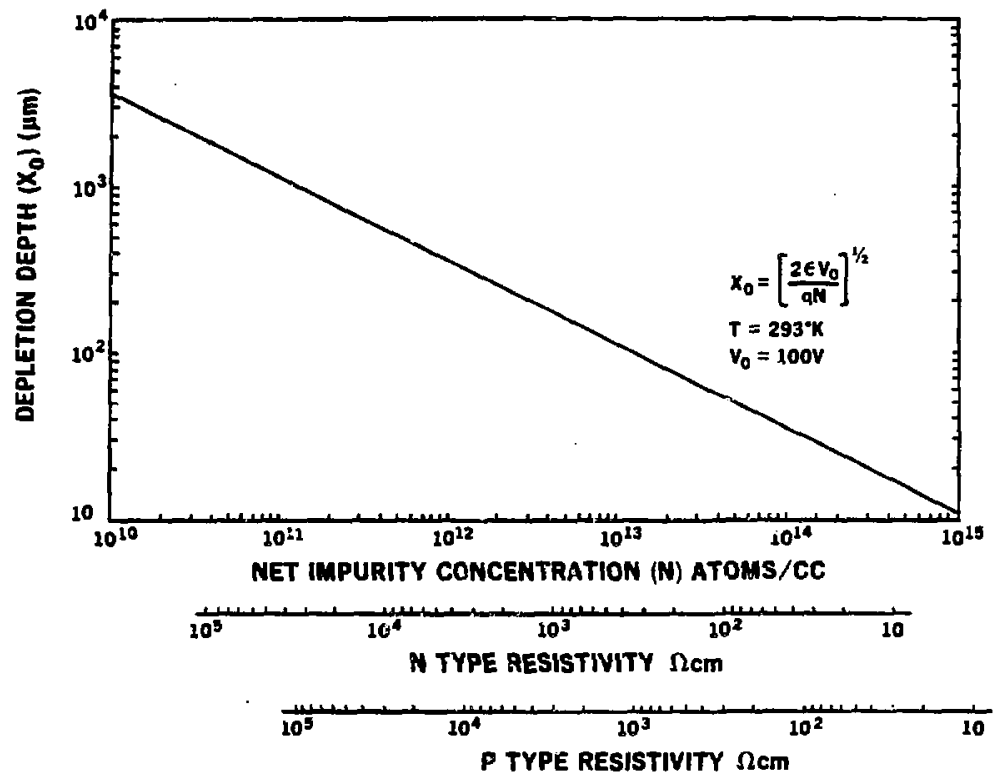

XIL $2210+274$

Fig. 5. Depletion width (um) with 100 volts reverse bias as a function of the net impurity concentrations (atoms/cc). Also shown are the n-type and p-type crystals' resistivities corresponding to the impurity concentration.

\section{DLFFUSED}

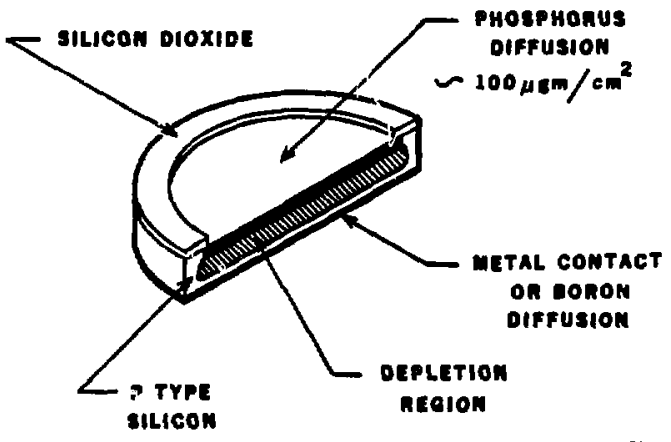

Fig. 6. Schematic of a diffused junction detector. 


\section{IOA IMYLANTED}

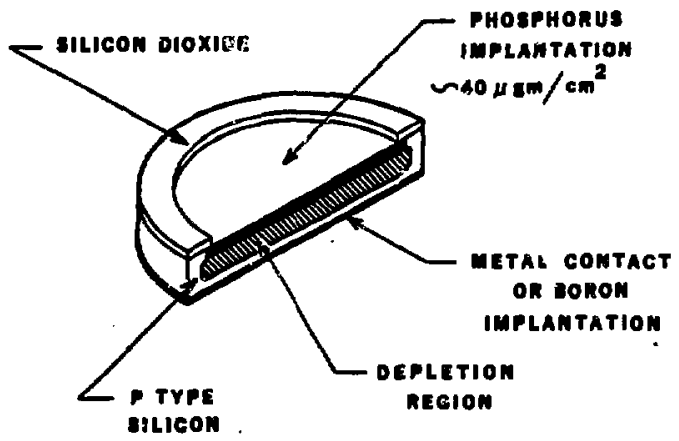

X86 792-8273

Fig. 7. Schematic of an ion-implanted junction detector.

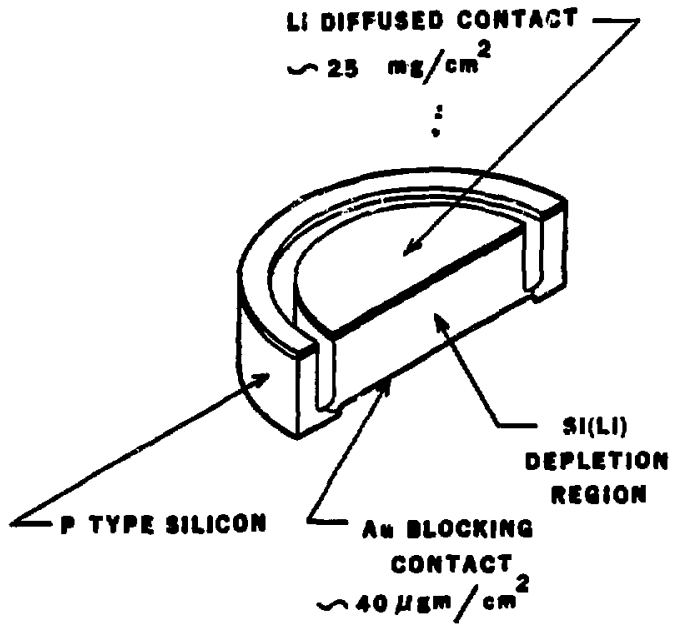

XEL 792-8269

Fig. 8. Schematic of a lithium-ion compensated detector. 


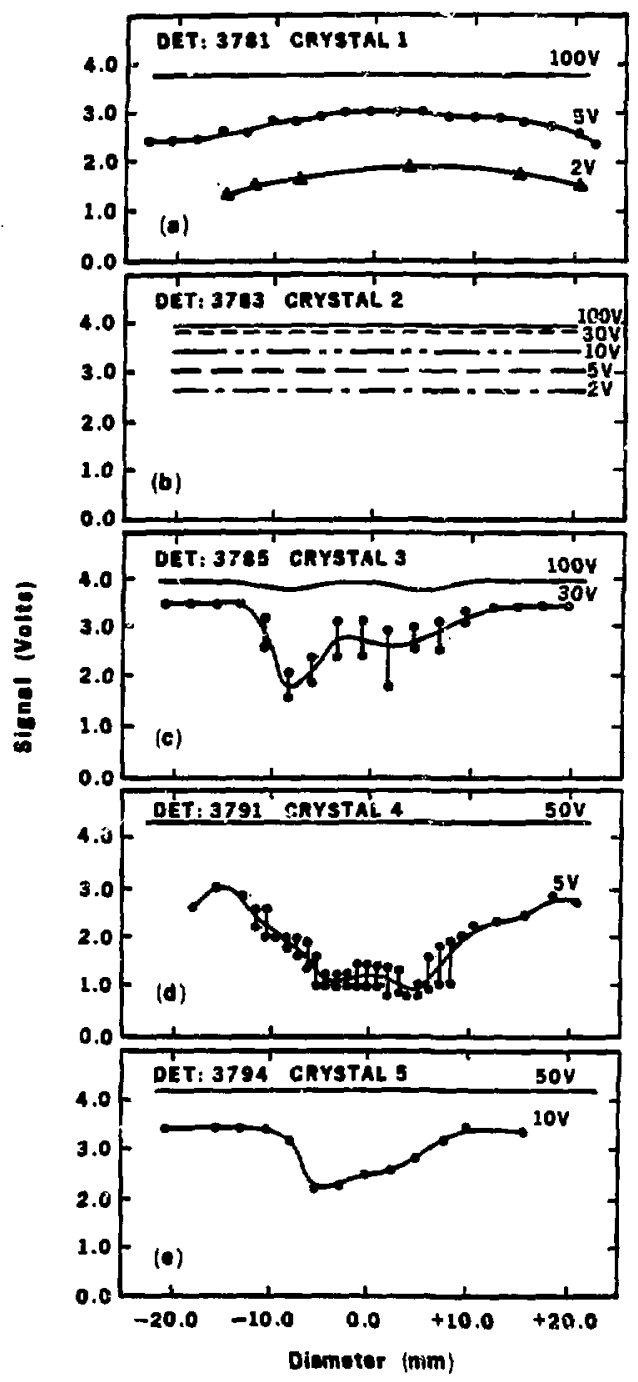

X8L $813-8332 \mathrm{~A}$

Fig. 9. The alpha particle scans of detectors made from five different crystals with various applied voltages. The lines connecting points in (c) and (d) indicate the presence of multiple peaks. 


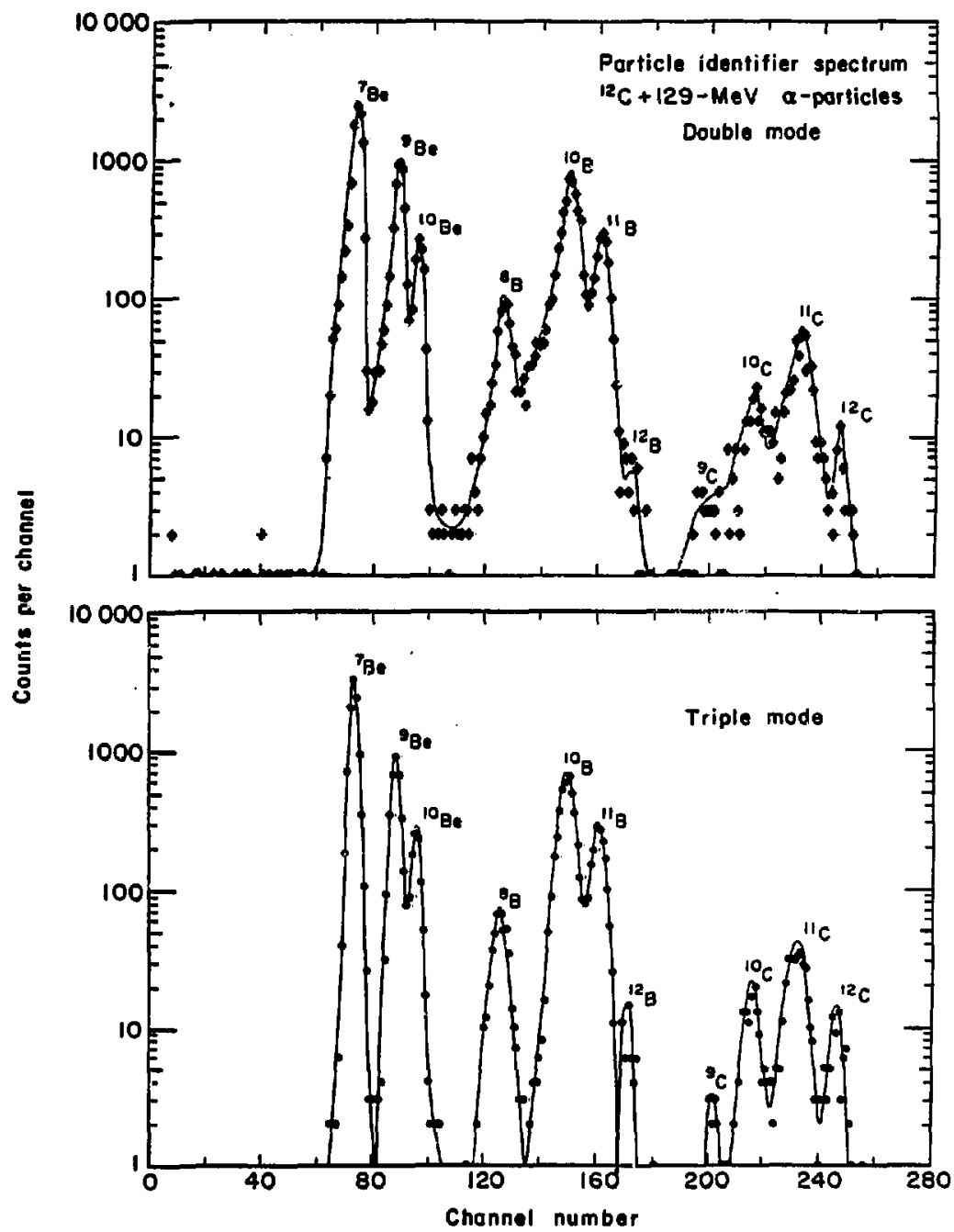

XBL 741-185

F19. 10. The particle identification spectrum produced by (a) a dual element $A E_{1} E$ telescope and $(b)$ a triple element $\triangle E, \triangle E, E$ telescope on the same $12 \mathcal{C}+129$ ineV alpha experiment. 
Fig. 11. Prototype vertex detector

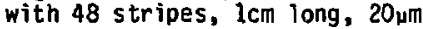
wide and $60 \mu \mathrm{m}$ apart. on ly 40 of the stripes are connected by aluminum wires which are potted in epoxy to improve the device ruggedness.

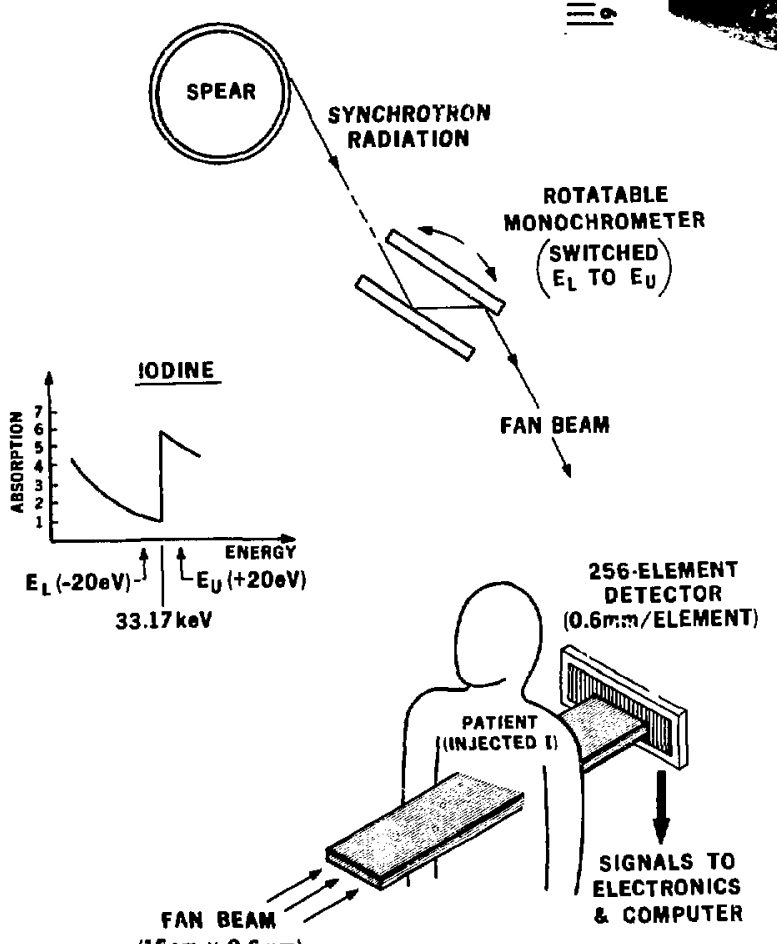

Fig. 12. Pictorial representation of the system for performing angiography using synchrotron radiation. 


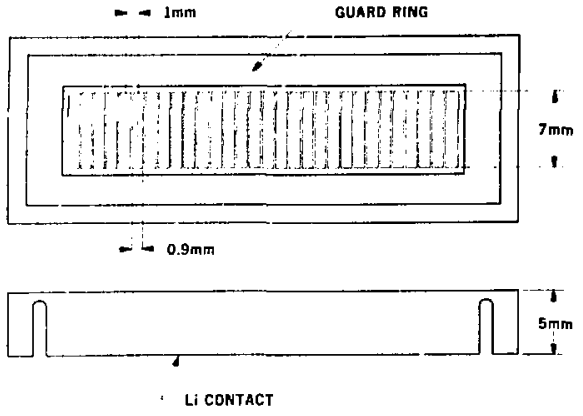

GEOMETRY
Fig. 13. Geometry of the 30-element silicon lithium-ion compensated detector used to procuce the images in Fig. 14.

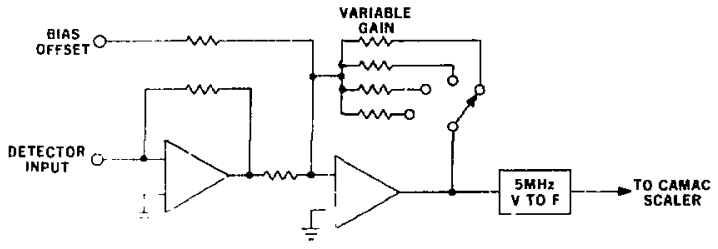

ELECTRONICS

MULTIELEMENT Si'Li) DETECTOR

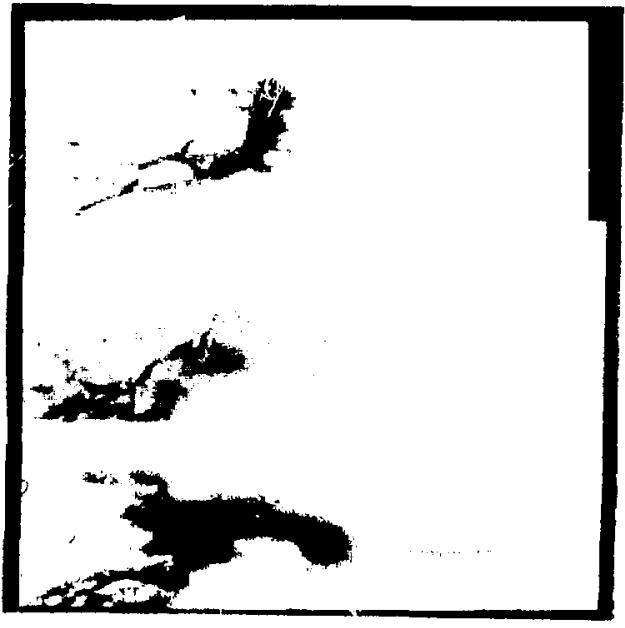

Fig. 14. Angiographs of blood flow in a dog's heart. The pictures are at two-second intervals after the injection of iodine with a catheter (top frame). 